\title{
Influenza A or COVID-19 Reinfection? Making the Right Diagnosis during the COVID-19 Pandemic
}

\author{
Njideka Udochi, MD, MPH, FAAFP, AAHIVS*, Hazel Jones Parker, DNP, CRNP, AAHIVS and \\ Melonie Owusu, BSN, RN
}

Millennium Family Practice in Columbia, Maryland, USA

*Corresponding author: Njideka Udochi, MD, MPH, FAAFP, AAHIVS, Millennium Family Practice in Columbia, 8900 Columbia 100 Pkwy Suite G, Columbia, MD, USA, 21045, 410-740-9001

\author{
Keywords \\ COVID-19, HIV, Influenza A, COVID-19 co-infection, \\ Healthcare workers, Infectious disease, Antiretrovirals
}

\section{Introduction}

As the second wave of SARS-CoV-2 (COVID-19) rages through the Unites States (US), it is coinciding with Influenza season, both being spread via respiratory droplets. Influenza symptoms such as cough, myalgia, and fever mirror symptoms of COVID-19.

Reinfection with the SARS-CoV-2 virus has been described but is relatively rare [1]. Several studies have described patients with long periods of positivity after initial COVID-19 infections. Disrupted care during the COVID-19 pandemic may contribute to increased susceptibility to viral diseases including COVID-19 in People living with HIV (PLWH). COVID-19 protective practices such as telemedicine and mail-order pharmacies may lead to gaps in care and poorer adherence in some patients $[2,3]$. Subsequently, increased viral load (VL) may also increase the risk for viral infections including COVID-19 and Influenza. Reports of false-positive results in patients diagnosed with COVID-19 are not common but have been described in recent studies. Technical issues including contamination during sampling and cross-reactions with other viruses could be responsible for false-positive results $[4,5]$.

In this case report, we present an unsuppressed patient living with HIV diagnosed and treated for COVID-19 who presented with similar symptoms 138 days later and was re-diagnosed with COVID-19 after testing negative in the interim and found to be Influenza $A$ positive and COVID-19 negative with a repeat reverse-transcriptase-polymerase chain reaction (RT-PCR) test.

\section{Case Report}

Ms. $R$ is a 51-year-old African American single mother who works as a medical technician in a local hospital. She has been a patient at a suburban family practice for fifteen years. Her past medical history includes Hypertension, HIV, Obesity, Prediabetes, Hyperlipidemia, and Vitamin $D$ deficiency. All these comorbidities were controlled with medications and/or lifestyle modifications at the time of her initial infection with SARS-CoV-2 including an undetectable HIV viral load for the last 15 years.

In mid-July of 2020, the patient was diagnosed with COVID-19 via RT-PCR testing at the hospital where she is employed. She immediately followed up with her Primary Care Provider (PCP) and was found to be symptomatic with a fever of $102 \mathrm{~F}$, sore throat, and nasal congestion. She denied shortness of breath and reported taking only Tylenol with minimal relief of fatigue, cough, and myalgia. She was prescribed Augmentin (Amoxicillin Clavulanic Acid) $875 \mathrm{mg}$ bid for ten days, Tessalon Perles (Benzonatate $100 \mathrm{mg}$ po bid $\times 5$ days, zinc 220 $\mathrm{mg}$ po qd, vitamin C $500 \mathrm{mg}$ po qd, and Aspirin $81 \mathrm{mg}$ po qd. She continued single tablet Biktarvy (bictegravir/ emtricitabine/tenofovir alafenamide) 1 tab qhs for antiretroviral treatment. The patient's VL was undetectable $<40$ copies per millimeters $(\mathrm{mL})$ at this time. Her statin therapy and vitamin D3 50000 iu q week replace- 


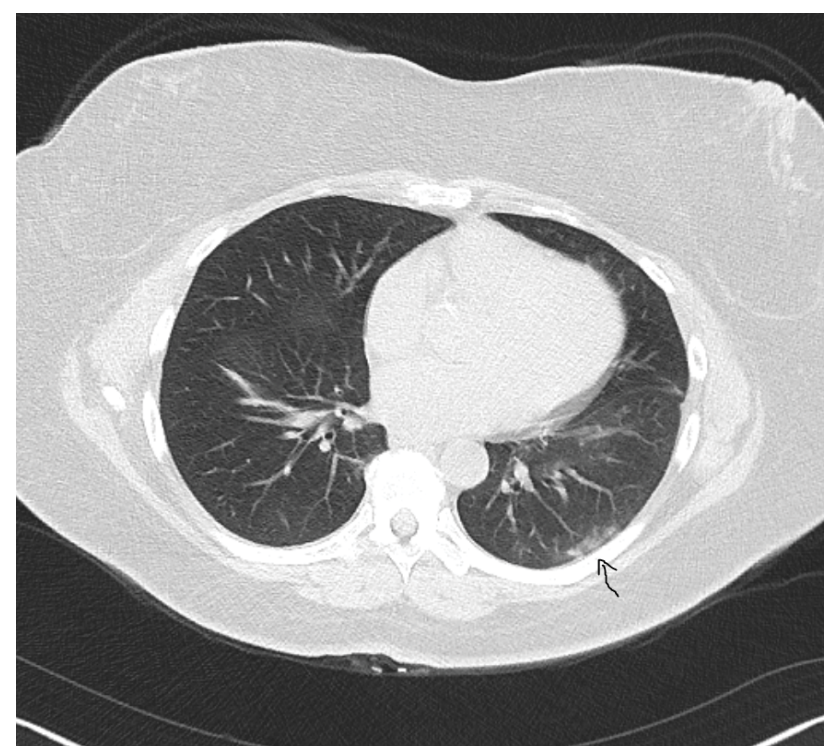

Figure 1: Evidence of diffuse ground glass opacities peripheral and globular in appearance within both lower lobes. This finding is compatible with COVID-19 pneumonitis.

ment were also continued at this time. She was also educated on the CDC guidelines for self-quarantine and symptoms that warranted escalation of care. After continued complaints of cough and new-onset of shortness of breath eight days later, a Chest Computed tomography (CT) scan was obtained and found to be consistent with COVID-19 pneumonitis displaying diffuse groundglass opacities within both lower lobes which were peripheral and globular in appearance (Figure 1). The patient was seen via telemedicine five days later and was found to be improving except for continued fatigue. Antibiotic therapy via Levaquin (Levofloxacin) $500 \mathrm{mg}$ a day by mouth for ten days was completed, and the patient continued zinc, vitamin $C$, vitamin $D$, and aspirin. A repeat CT scan order was written for 6 weeks and the SARS-CoV-2 RT-PCR was repeated and was negative.

The patient had a follow-up telemedicine visit in Mid-August of 2020 and she reported completion of her COVID-19 treatment and denied any of her previous symptoms. Routine management of her comorbidities including HIV continued, however, when the patient attempted to get her antiretrovirals refilled she was mandated to switch to a mail-in pharmacy and never received her prescription.

During an early December telemedicine appointment, the patient, a medical technician working with COVID-19 patients in a local hospital, reported a second positive SARS-CoV-2 RT-PCR test following exposure in her place of employment. Symptoms at the time of visit included fatigue and sore throat. She denied nausea, vomiting, diarrhea, shortness of breath, myalgia, and loss of taste or smell. The patient was not taking any over the counter medications at that time. She was again educated on the current CDC self-quarantining guidelines and placed on Augmentin (Amoxicillin Clavulanic Acid) $875 \mathrm{mg}$ twice a day by mouth for ten days for

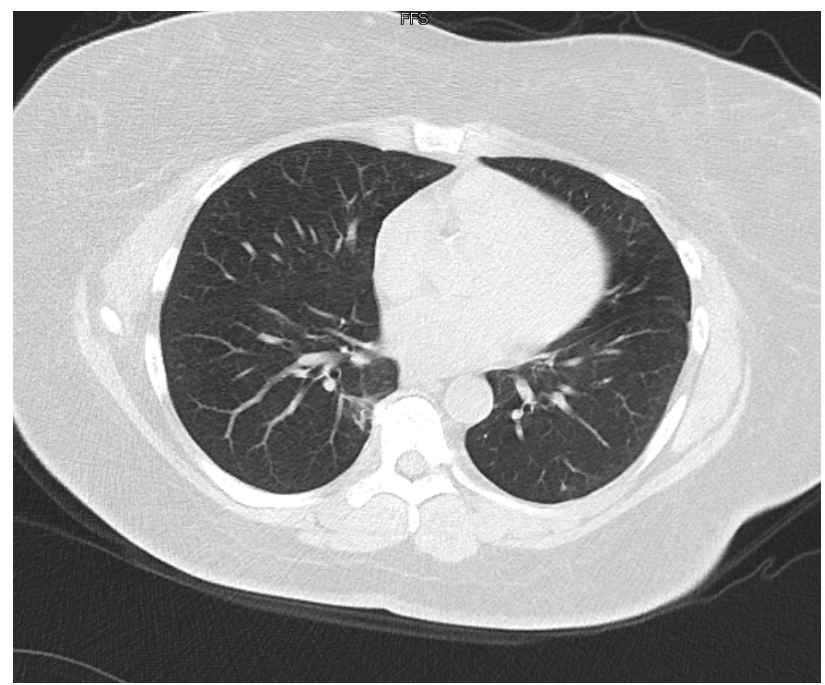

Figure 2: Interval significant improvement and decrease in bilateral ground glass infiltrates. Results show COVID-19 pneumonitis is essentially resolved. There are also areas likely to be atelectasis evidence by slightly increased irregular parenchymal opacities in the medial aspect of the right lower lobe and tracking along the prominent adjacent thoracic vertebral body osteophytes.

antibiotic therapy, vitamin c, zinc, aspirin, and continued her vitamin D supplements.

During a telemedicine follow-up visit the next day, the patient reported being off of her antiretrovirals since the end of August. Her symptoms had worsened now including shortness of breath, fever, chills, fatigue, and cough. She generally felt very ill stating she feels "worse than last time". Additional therapies including high dose vitamin D with Calcium, prednisone, and albuterol therapy were initiated. She was given samples of her antiretroviral regimen until she filled the prescription.

The repeat CT scan (Figure 2) showed no groundglass opacities consistent with COVID-19.

An attempt to get genomic testing from the hospital lab was futile as the specimen had been discarded so another repeat RT-PCR and respiratory pathogen panel (RPP) was repeated 3 days following the positive RT-PCR and was shown to be negative for COVID-19 but positive for Influenza A and Staph Aureus. She reported receiving the Influenza vaccine at her job on 11/1/2020. The patient was started on Oseltamivir (Tamiflu) $75 \mathrm{mg}$ twice a day for 5 days to treat the Influenza $A$ and reported improvement in her symptoms $48 \mathrm{hrs}$ later. Lab work ordered at this visit included a CD4 count, HIV viral load, CBC, CMP, CRP Cardio, CRP Quant, D-Dimer, LDH, Vitamin $D$, and Ferritin levels. Abnormal results included a detectable viral load at 480 copies/L, an elevated CRP Cardio (3.05 mg/L), elevated LDH (255 IU/L), and elevated Ferritin $(195 \mathrm{ng} / \mathrm{mL})$. Though she had an elevated CRP Cardio, LDH, and Ferritin, there was no evidence of significant cardiovascular events. Her CD4 count was 689 in comparison to her previous level which was 1412 
in previous months. These labs were drawn a week after she was re-started on antiretroviral therapy as she was not able to draw her labs before this. It can be inferred the start of antiretroviral therapy caused her viral load and CD4 count to be altered not giving a true depiction of her levels while completely off therapy during the past four months.

\section{Conclusion}

Influenza and COVID-19 co-infection can occur in patients and can present with similar symptoms $[4,6]$. Although the incidence of Influenza has declined this year, it is essential to recognize other viral diseases can still occur during the current pandemic. Correctly diagnosing Influenza results in early treatment, ameliorating symptoms and preventing complications such as Influenza pneumonia. When the diagnostic thought process is not open and independent to other diagnoses aside from COVID-19, a missed diagnosis or delay in treatment occurs [4].

Though the occurrence of COVID-19 reinfection is possible, it is not common as evident in this case report. The second positive test occurred 138 days after the initial infection. Obtaining a respiratory panel in addition to COVID-19 RT-PCR testing will aid in the diagnosis and management of vulnerable populations including PLWH, minorities, and health care workers. It is also important to assess for cardiovascular disease and events in the presence of both COVID-19 and Influenza [4]. The burden from these viruses on cardiovascular health increases the risk of hospital mortality and the increased utilization of health care resources [4]. Encouraging vaccinations for Influenza at this time will also help to further decrease its incidence. Once Influenza is diagnosed, prompt treatment can help to decrease COVID-19 morbidity as evidence shows Influenza treatment has led to improved outcomes and aided in quick recovery [6]. In addition, encouraging adherence to antiretrovirals in PLWH will lead to viral suppression and decrease the likelihood of infections with viruses such as Influenza $A$ or $B$ as well as COVID-19. Though COVID-19 reinfections and co-infection are both rare, each should be considered and ruled out to encourage improved patient outcomes especially amongst vulnerable populations $[1,6]$.

\section{Author Disclosure}

Hazel Jones Parker is on the speaker's bureau for Janssen and Gilead.

\section{References}

1. Surkova E, Nikolayevskyy V, Drobniewski F (2020) False-positive COVID-19 results: Hidden problems and costs. Lancet Respir Med 8: 1167-1168.

2. Kalichman SC, Eaton LA, Berman M, Kalichman MO, Katner H, et al. (2020) Intersecting Pandemics: Impact of SARS-CoV-2 (COVID-19) Protective Behaviors on People Living With HIV, Atlanta, Georgia. J Acquir Immune Defic Syndr 85: 66-72.

3. Mugavero MJ, Westfall AO, Cole SR, Geng EH, Crane HM, et al. (2014) Beyond core indicators of retention in HIV care: missed clinic visits are independently associated with all-cause mortality. Clin Infect Dis 59: 1471-1479.

4. Agarwal MA, Ziaeian B, Lavie CJ, Fonarow GC (2020) Cardiovascular Disease in Hospitalized Patients With a Diagnosis of Coronavirus From the Pre-COVID-19 Era in United States: National Analysis From 2016-2017. Mayo Clin Proc 95: 2674-2683.

5. Ozaras R, Cirpin R, Duran A, Duman H, Arslan O, et al. (2020) Influenza and COVID-19 coinfection: Report of six cases and review of the literature. J Med Virol 92: 26572665.

6. Konala VM, Adapa S, Gayam V, Naramala S, Duggubati SR, et al. (2020) Co-infection with influenza $A$ and COVID-19. Eur J Case Rep Intern Med 7: 001656. 